\title{
La transparence un principe ascendant de la réalisation de l'Union Européenne
}

Loïc Grard

\section{(2) OpenEdition}

12 Journals

Édition électronique

URL : http://journals.openedition.org/communicationorganisation/2351

DOI : 10.4000/communicationorganisation.2351

ISSN : $1775-3546$

Éditeur

Presses universitaires de Bordeaux

Édition imprimée

Date de publication : 1 mai 2000

ISSN : 1168-5549

Référence électronique

Loïc Grard, «La transparence un principe ascendant de la réalisation de l'Union Européenne », Communication et organisation [En ligne], 17 | 2000, mis en ligne le 27 mars 2012, consulté le 01 mai 2019. URL : http://journals.openedition.org/communicationorganisation/2351 ; DOI : 10.4000/ communicationorganisation.2351

Ce document a été généré automatiquement le 1 mai 2019.

(c) Presses universitaires de Bordeaux 


\title{
La transparence un principe ascendant de la réalisation de l'Union Européenne
}

\author{
Loïc Grard
}

1 L'interdisciplinarité a ceci de fécond qu'elle aide chacune des composantes intégrées à réfléchir sur les orientations qu'elle doit choisir quant à son objet d'étude. Lorsque le juriste intéressé au droit européen vient s'aventurer dans la science de l'information et de la communication, il y découvre pour sa matière des impératifs qui ont chacun leur logique propre. Améliorer la communication permet d'appréhender un processus organisant de l'Union européenne, facteur essentiel au sens propre, à prendre en considération pour un droit qui reste un droit de la construction de l'identité européenne. Informer toujours mieux permet d'asseoir l'Europe comme réalité autre que géographique et ainsi d'accroître sa crédibilité comme organisation superposée aux États.

2 Ces perspectives complémentaires rencontrent l'Union européenne qui demeure aujourd'hui un objet juridique non identifié, ne pouvant être déjà apparentée ni à une fédération, ni à une confédération, ne pouvant plus être assimilée à une organisation internationale. Autrement dit, l'Union européenne est en train de s'imposer comme une organisation d'un genre juridique nouveau. Une nouvelle forme d'organisation est en train de s'inventer sous nos yeux par la volonté d'une quinzaine d'États. C'est pourquoi, afin de contribuer à la compréhension de ce processus atypique, il apparaît digne d'intérêt de réunir les perspectives de la science juridique et de la science de l'information et de la communication. Le point de départ de cette étude conciliant des préoccupations complémentaires passe en conséquence par une question simpliste mais incontournable!

3 La communication et l'information pour l'affirmation de l'identité de l'Union européenne auprès des citoyens sont-elles suffisantes? On peut en douter, si on analyse les raisons pour lesquelles un européen sur deux boude les élections au Parlement européen, qui tiennent soit à une mauvaise connaissance de l'institution (le Parlement européen, à quoi ça sert ?), soit à une connaissance erronée (le Parlement européen, ça ne sert à rien !... $)^{1}$. 
Pour corriger ce hiatus entre l'institution et le particulier, le droit développe notamment le respect du principe de transparence ${ }^{2}$. Le principe ne s'est pas imposé ex nihilo. Il est monté en puissance depuis l'adoption du traité de Maastricht en 1992. Successivement, le Sommet des chefs d'État et de gouvernement, à Édimbourg en décembre 1992, met l'accent sur une Communauté proche de ses citoyens, l'accord interinstitutionnel du 25 octobre 1993 exprime l'intention commune du Conseil de l'Union européenne, de la Commission européenne et du Parlement européen sur la démocratie, la transparence et la subsidiarité. Plus récemment, le traité d'Amsterdam modifie l'article $1^{\mathrm{er}} \mathrm{du}$ traité sur l'Union européenne pour y inscrire que «les décisions sont prises dans le plus grand respect possible du principe d'ouverture... »,principe qui devient un des éléments du caractère démocratique de la prise de décision dans l'Union européenne ${ }^{3}$.

5 La Communauté et l'Union européennes rencontrent donc de manière évidente et nécessaire la prétendue " troisième génération des droits de l'Homme ». Après avoir consacré les droits économiques dès sa création, renforcé le contrôle des droits fondamentaux individuels à partir du début des années soixante-dix, le droit de savoir, le droit à l'information font irruption dans la relation entre le citoyen européen et l'administration européenne. Ce nouvel impératif démocratique à la construction européenne s'impose depuis le début des années 1990, une dizaine d'années après que la France se fut engagée dans un grand mouvement de transparence entre administration et administrés4.

En France, les manifestations de ce mouvement sont devenues particulièrement visibles avec la création de la Commission d'accès aux documents administratifs (CADA), de la Commission national informatique et libertés (CNIL) ou encore la multiplication des centres interministériels de renseignement administratif (CIRA). Pour l'Union européenne, la transparence n'a pas encore rencontré des effets structurants aussi connus et reconnus. Au contraire, un feu nourri de critiques s'abat régulièrement sur la construction européenne pour en stigmatiser l'opacité. La démission récente de la Commission a notamment pour raison la condamnation de certains de ses comportements jugés occultes. Pourtant, les initiatives sont nombreuses pour améliorer l'accès du public à l'information communautaire (I), ainsi que son accessibilité (II).

\section{L'accès du public à l'information communautaire}

7 Une déclaration annexée au traité sur l'Union en 1992 à Maastricht relative au droit d'accès à l'information "estime que la transparence du processus décisionnel renforce le caractère démocratique des institutions, ainsi que la confiance du public envers l'administration". Cette déclaration fait office de prudent contrepoids à un traité qui, dans sa version "Maastricht", reste extrêmement disert sur les questions de communication, de publicité ou d'information se rapportant à la Communauté. Y figurent notamment des dispositions sur la confidentialité : les membres des institutions sont tenus de ne pas divulguer les informations couvertes par le secret professionnel (article 214). Seul l'article 191 fait obligation de publier les actes juridiques de la Communauté au Journal officiel des Communautés européennes (ci-après JOCE). L'information se confond donc avec l'information officielle. Derrière ces dispositions, la pratique et quelques fois le droit ont engendré des progrès aussi bien quant à l'information sur la Communauté qu'aux informations détenues par la Communauté. 


\section{L'information sur la Communauté}

8 L'information sur la Communauté a pour objet de réduire la distance avec les particuliers. Faire en sorte que le fonctionnement, comme les activités de la Communauté ne se développent pas à l'insu du citoyen constitue assurément une exigence démocratique.

\section{Les activités de la Communauté}

9 La Communauté européenne a toujours mené une politique de communication et d'information sur ses activités dont le développement est lié au succès croissant du besoin de transparence ${ }^{5}$. C'est pourquoi, les techniques retenues se sont diversifiées et perfectionnées, notamment depuis que l'objectif de la réalisation du marché intérieur a été posé. Désormais, l'information porte autant sur ce qui va être fait que sur ce qui est fait.

10 Par exemple, le projet se rapportant à la réalisation du marché intérieur au $1^{\mathrm{er}}$ janvier 1993 a été d'abord inscrit dans un livre blanc dont la publication en 1985 a été le moyen de susciter un dialogue élargi autour de la prévisibilité de l'action communautaire. Cette méthode s'est par la suite généralisée par la publication régulière de livres blancs ou livres verts ou autres mémorandums se rapportant au développement de certaines politiques. Elle permet de recueillir tout avis pertinent en vue de façonner la législation. Ici, le rapport avec le "lobbying» se scelle et permet de faire redescendre l'information vers les intéressés et d'en attendre le «feed-back».

11 En ce qui concerne le renseignement sur ses activités, la Communauté emploie des techniques multiples. Les énumérer reviendrait à faire un inventaire à la Prévert. On peut toutefois souligner la priorité accordée à l'information aux entreprises par la constitution du réseau des Euro-info-centres, y compris dans les Pays d'Europe centrale et orientale. L'accent est mis également en direction du grand public avec la diffusion de documents de vulgarisation, notamment par les offices de publication des Communautés européennes.

Il est loisible d'observer que les mesures adoptées n'ont pas pour objet de multiplier l'information délivrée au grand public, mais de la rendre plus compréhensible. La doctrine affichée n'est pas de plus informer, mais de mieux informer. Ainsi la relation avec les médias a été améliorée en raison d'un service de presse disponible 24 heures sur 24. Les techniques modernes d'information sont privilégiées avec notamment la constitution du site Internet «Europa " permettant de s'orienter vers les activités des différents services et institutions. Des actions de sensibilisation du grand public sont menées; les plus visibles se sont rapportées à la mise en place du marché intérieur et de la monnaie unique. Enfin, la Communauté a mis en place « Eurobarométre », en vue d'une évaluation permanente de l'efficacité et du rapport coûts-avantages des activités d'information et de communication.

La communication passe aussi par des techniques plus sophistiquées encore. À cet égard, force est de souligner le soin avec lequel sont choisis les sigles pour désigner les programmes d'action communautaires. Pour corriger l'hermétisme, un minimum de correspondance est garanti entre le contenu et le contenant des sigles. Par exemple désigner par "SOCRATES" les programmes de mobilité étudiante permet de faire connaître et reconnaître l'action entreprise. 
14 Mais l'information sur la Communauté ne doit pas être l'exclusivité de la Communauté. Cette mission incombe également à ses États membres. Plus exactement, il y ici une mission qui doit être exercée par les autorités publiques y compris de niveau infra étatique. Cet impératif doit d'abord être vérifié dans la transparence de la mise en œuvre des politiques communautaires. On pense, entre autre exemple, à l'action des Fonds structurels dans les régions dont la récente réforme rappelle aux États leurs obligations en termes d'information et de communication. Mais il pèse également sur les États membres de participer à l'information sur la Communauté. À cet égard des mécanismes se mettent en place entre responsables de l'information dans les États et les institutions européennes pour échanger leurs expériences et mettre en œuvre un système de communication mutuelle et consultation concernant l'information sur l'Union.

de ces quelques observations sur l'information se rapportant à l'activité, une impression d'empirisme prévaut. Cela n'a rien d'étonnant, eu égard au fait que les assises juridiques de la politique d'information apparaissent incertaines. Des événements récents l'ont rappelé.

16 À la fin de l'année 1998, un rapport de la Cour des comptes a stigmatisé l'absence de cadre réglementaire suffisamment précis pour justifier le financement de nombre d'activités d'information menées par la Commission européenne. Comme symbole de cette situation, a été mis en avant l'outil audiovisuel de promotion de l'Union européenne et de ses États membres dans les pays tiers : "Contact ${ }^{6}$. Il s'agit d'une coproduction entre d'une part, les sociétés de radiodiffusion et les associations culturelles nationales et d'autre part le service audiovisuel de la Commission. Le tout est réalisé dans un cadre tout à fait informel auquel sont consacrés 70000 euros par an. On reproche notamment à cette initiative d'être devenue un moyen de propagande aux mains de la seule Commission européenne qui y exerce un dirigisme informationnel. Cette critique fait nettement apparaître un débat de fond quant à la maîtrise de l'information au sein de l'Union entre ses composantes.

17 C'est pourquoi le Conseil de l'Union a rendu publiques des conclusions visant à ce qu'il contribue plus activement à la politique de l'information en contrepoint de la Commission et du Parlement européen ; ce qui ne peut que contribuer à assurer une plus grande transparence de ses activités vis-à-vis des citoyens européens. Cela revient à diversifier les sources d'information pour en valoriser la qualité : éviter une pensée unique de la Commission sur l'Union européenne. C'est pourquoi, désormais le Conseil développe des moyens électroniques d'information à la disposition des journalistes comme du grand public. Ainsi depuis le $1^{\text {er }}$ janvier 1999, peut-on consulter un registre public des documents du Conseil accessible par le réseau Internet. Ces évolutions font apparaitre un mouvement vers une « démonopolisation » de la politique de l'information. Mieux informer, c'est aussi diversifier la source de l'information.

\section{Le fonctionnement de la Communauté}

18 La transparence s'attache aussi au fonctionnement des institutions de la Communauté. C'est ainsi que depuis sa révision du 6 décembre 1993, le règlement intérieur du Conseil contient des prescriptions permettant de garantir la transparence du processus décisionnel menant à l'adoption d'actes de portée obligatoire. Cet impératif qui correspondait à une initiative propre au Conseil a été conforté avec le traité d'Amsterdam qui inscrit dans le traité $\mathrm{CE}$ une disposition indiquant que «lorsque le Conseil agit en sa 
qualité de législateur, les résultats et les explications des votes, ainsi que les déclarations inscrites au procès-verbal, sont rendus publics » (article $208 \$ 3$ ). Le nouveau règlement intérieur du Conseil arrêté le 31 mai 19997, en vigueur depuis le premier juillet de la même année, fixe également le régime de l'information préparatoire de ses réunions, ainsi que de la publicité des débats.

La transparence des travaux du Conseil commence par la fixation de l'ordre du jour des sessions. En fait, le règlement intérieur prévoit un ordre du jour provisoire de manière à informer les États membres et la Commission, le plus complètement, au moins quatorze jours à l'avance; cela pour leur permettre de préparer la session et de ne pas être mis devant le fait accompli. Il s'agit ici d'une transparence interne, pour une circulation et une diffusion de l'information entre les détenteurs du pouvoir politique dans la Communauté. Dans le cadre ainsi tracé, les intéressés doivent recevoir toute la documentation se rapportant à chaque point de l'ordre du jour. Ce dernier devient définitif au début de chaque session et ne peut comporter un point supplémentaire que si les quinze membres se sont prononcés favorablement en ce sens. Mais si l'information est une condition de fonctionnement interne elle l'est également devenue au plan externe en raison de l'exigence de transparence envers le grand public.

Cette exigence doit quand même être nuancée à la lecture de l'article $4 \S 1$ qui postule que «Les sessions du Conseil ne sont pas publiques... ». Toutefois les débats d'orientation sur le travail semestriel du Conseil ou le programme annuel de la Commission font l'objet d'une retransmission publique par les moyens audiovisuels. Au cas par cas, il peut être décidé à l'unanimité d'étendre cette publicité aux questions les plus importantes pour l'intérêt de l'Union.

De même, l'article 5 \$ 1 érige en principe le fait que les délibérations relèvent du secret professionnel. Cette confidentialité se justifie par un souci d'efficacité. Le Conseil est un lieu de compromis et de discussions. Si toutes les positions exprimées ainsi que les changements de positions en vue de favoriser l'entente entre les quinze États membres étaient rendus publiques, les négociateurs pourraient se trouver gênés car moins libres de leurs initiatives. Ici, le besoin de transparence et de démocratie est contrebalancé par le souci d'efficacité. Toutefois, dans les hypothèses où le Conseil agit ès qualité de législateur, les résultats et les explications des votes, ainsi que les déclarations inscrites au procès-verbal sont rendus publics, qu'il s'agisse d'une position commune ou de l'adoption définitive d'un texte. De cette manière, la position de chaque État membre de la Communauté peut être clairement identifiée.

Le Conseil n'est pas la seule institution dont le fonctionnement évolue vers la transparence. Le Parlement européen a également modifié son règlement intérieur en ce sens le 11 mars 1999. L'exemple le plus significatif de l'effort accompli réside dans l'abolition de la possibilité de procéder à des débats à huis clos sur proposition des deux tiers des membres. La Commission est en train de suivre le même chemin en raison de la modification de sa structure administrative suite à la démission collective du mois de mars 1999. Son nouveau président, Romano Prodi, a promis la transparence lors de son audition par le Parlement européen, préalable à sa nomination définitive

23 En tout état de cause, l'exemple du Conseil nous montre que dans son fonctionnement, la Communauté européenne concilie inévitablement impératif d'efficacité et principe de transparence. Ce dernier doit composer avec des contraintes externes, en ce qui concerne 
l'accès à l'information détenue par la Communauté. La transparence est maximale, elle ne peut être totale.

\section{L'information détenue par la Communauté} conduit des travaux dont les résultats ont été publiés dans une communication sur «L'accès du public aux documents des institutions ${ }^{8}$, qui a notamment pour finalité d'informer sur l'état des lieux et des projets. La transparence se prépare dans la transparence et fait apparaître une situation de confidentialité trop systématique de l'information détenue par la Communauté. l'information détenue par la Communauté. Mais sur ce point, son droit se singularise par une double orientation. Sur des questions particulièrement sensibles, le droit à se voir informé a été consacré (par exemple l'examen des comportements concurrentiels des entreprises). Dans les autres domaines, on peut aujourd'hui prétendre qu'il y a un droit du citoyen européen qui est en train de s'affirmer. Rompre avec la culture du secret est en train de devenir un impératif pour restaurer la confiance du public dans la démocratie au niveau européen.

\section{Un droit d'accès du public initialement ponctuel}

\section{L'exemple du droit de la concurrence}

Sur la base des articles 85 et suivants du traité CE, la Commission est investie de pouvoirs en vue de faire respecter par les entreprises le droit de la concurrence. Elle peut à ce titre interdire des comportements et prononcer des sanctions sous forme d'amendes extrêmement lourdes. Sans être des procédures pénales, ces méthodes apparaissent "pénalisantes ». C'est pourquoi, les droits de la défense des entreprises contrôlées méritent une protection maximale qui passe par le respect de la transparence. La pratique décisionnelle de la Commission et la jurisprudence du juge communautaire ont fait apparaître, à ce titre, des principes qui ont été codifiés par une communication de la Commission du 27 janvier 19979. L'examen de ceux-ci fait apparaître les grandes orientations que doit respecter le droit d'accès à l'information détenue par la Communauté. La concurrence fait donc figure de précédent.

Le droit d'accès au dossier forme la première déclinaison de la transparence en matière de contrôle sur les entreprises : «La Commission... divulgue aux entreprises en question tous les faits, circonstances, ou documents sur lesquels elle se base, pour permettre de faire connaître utilement leur point de vue $»^{10}$. Cela signifie a contrario que la Commission est fondée à refuser la divulgation d'informations ne lui étant d'aucune utilité pour fonder sa décision. Il s'agit d'un droit d'accès fonctionnel, visant à mettre à égalité devant l'information l'entreprise et l'autorité administrative, dans le cadre d'une procédure contradictoire.

Mais il ne s'agit pas d'un droit d'accès à la totalité du dossier. Il demeure une réserve de confidentialité au profit de la Commission, dont les contours ont été notamment précisés dans un arrêt de la Cour de Justice en date du 6 avril $1995^{11}{ }^{1}$. Certains documents sont par nature non communicables car ils se rattachent aux relations avec la justice. D'autres sont manifestement non communicables, notamment les informations saisies dans une 
entreprise tierce ou les correspondances avec celle-ci. Donc la Commission peut opposer dans une certaine mesure le secret à l'entreprise soumise à son contrôle.

Il en va de même de l'entreprise en question qui peut opposer la confidentialité de ses informations à la Commission. En premier lieu, elle peut refuser de divulguer certaines informations comme, par exemple, un échange épistolaire avec un avocat ${ }^{12}$. En second lieu, elle a un intérêt légitime à ce que ses secrets d'affaire ne soient pas divulgués par la Commission, ni auprès d'entreprises tierces, ni auprès des États ${ }^{13}$. S'il en allait autrement, les procédures seraient déclenchées par la concurrence dans le seul but de recueillir des informations. On comprend ainsi que la transparence de l'information détenue par la Commission s'arrête là où commence la protection du secret des affaires. Les intérêts en présence doivent être mis en balance. Le droit de la concurrence démontre que l'accès aux documents détenus par la Communauté ne peut être un droit sans limite. Il doit composer avec l'obligation de secret professionnel pesant sur les autorités communautaires. Il peut être variable en fonction de la situation dans laquelle on se trouve $e^{14}$.

\section{Le cas particulier du droit de l'environnement}

La directive du Conseil n 90/313 du 7 juin 1990 relative à la liberté d'accès à l'information en matière d'environnement que détiennent les autorités publiques en vigueur depuis le 1 er janvier $1993^{15}$ est un cas particulier, car cette législation crée une obligation pesant sur les autorités compétentes des États membres. Toutefois, la Commission a affirmé lors de l'élaboration du texte que le principe s'auto-appliquerait aux institutions de la Communauté...

31 Un arrêt récent de la Cour de Justice des Communautés européennes ${ }^{16} e x p l i q u e ~ q u ' i l$ devait être donné une interprétation extensive à la notion d'information sur l'environnement et que les exceptions prévues par la directive sont soumises à interprétation restrictive. Il en ressort une claire volonté d'une transparence aussi large que possible exigée des États dans ce domaine.

\section{Un principe général du droit communautaire : l'accès du public aux documents communautaires}

Une réflexion conduite une quinzaine d'années plus tôt aurait mené à conclure sur le principe du secret ou de la confidentialité du fonctionnement interne des institutions communautaires, notamment pour le Conseil et la Commission. La situation a évolué dans le sens d'une affirmation progressive du droit d'accès des particuliers aux informations détenues par les instances de la Communauté ${ }^{17}$.

\section{L'émergence du droit d'accès aux documents détenus par les organes de la Communauté}

33 L'éclosion d'un principe général d'accès du public aux documents détenus par l'administration communautaire se manifeste comme le fruit d'une sédimentation juridique. La première influence provient des États membres, dont la plupart des réglementations consacrent de manière générale à titre de principe constitutionnel ou législatif le droit d'accès du public aux documents détenus par les autorités publiques. La seconde influence provient de la déclaration $n^{\circ} 17$ annexée au Traité sur l'Union depuis Maastricht relative au droit d'accès à l'information qui rattache ce droit au caractère 
démocratique des institutions. Une troisième influence est en provenance du médiateur européen qui voit de manière constante dans l'accès aux documents une contribution primordiale à la transparence, à la bonne gestion administrative et donc aux bonnes relations entre citoyens et administration communautaire. Enfin, le Conseil européen a invité de manière régulière au respect de cette exigence dans la relation entre la Communauté et le public. Cet ensemble de références comporte toutefois la faiblesse de ne pas être constitutif d'une règle de droit obligatoire à respecter.

Le législateur communautaire n'a pas adopté de réglementation générale sur le droit d'accès du public aux documents possédés par les institutions communautaires. Les seuls textes adoptés sont relatifs à l'auto organisation des institutions, qui arrêtent leur règlement interne de manière à préciser leur fonctionnement. Dans ce cadre, elles ont, au nom de la «bonne administration» adopté des mesures organisant l'accès des particuliers aux documents non publiés. De manière à mettre en cohérence leurs doctrines respectives, certaines ont énoncé conjointement un code de conduite ${ }^{18}$ qui fixe des principes, auxquels leurs règlements intérieurs sont fidèles.

Le code de conduite énonce un «principe général » selon lequel «Le public aura le plus large accès possible aux documents détenus par le Conseil ou la Commission ». On entend par document, tout écrit quel que soit son support, contenant des données existantes. Les orientations générales quant au traitement d'une demande sont fixées. Un certain nombre d'exceptions à la diffusion sont envisagées. Ce qui témoigne d'un droit à être informé qui n'est pas absolu. Ce qui est légitime, car ce droit doit être concilié avec celui d'être protégé par la confidentialité. il en va ainsi pour la protection de l'intérêt public, la protection de l'individu et de la vie privée, la protection des secrets commerciaux ou industriels, la protection des intérêts financiers de la Communauté, la protection de la confidentialité demandée par la personne dont l'information est originaire ou quelques fois du bon fonctionnement de l'institution.

Le code de conduite a servi d'orientation à la décision du Conseil $n^{\circ} 93 / 731 d u$ 20 décembre 1993 relative à l'accès du public à ses documents ${ }^{19}$. De même, le code de conduite a été mis en œuvre par la Commission par une décision $n^{\circ}$ 94/90 du 8 février $1994^{20}$ éclairée par une communication du 4 mars $1994^{21}$. En revanche, le code de conduite ne visait pas formellement les autres institutions et organes de la Communauté. Cela n'a pas empêché le Parlement européen de se mettre au diapason, certes tardivement, en prenant le 10 juillet 1997 une décision dans le prolongement de son règlement intérieur "relative à l'accès du public aux documents $"^{22}$. En outre, largement en raison de recommandations du Médiateur européen dans son rapport 1996, le Comité économique et social ainsi que le Comité des régions ont arrêté des décisions quant à l'accès du public à leurs documents, respectivement le 27 mai et le 17 septembre $1997^{23}$. Globalement les solutions arrêtées s'apparentent à celles du code de conduite ${ }^{24}$.

Le fait qu'il s'agisse d'actes se rapportant à l'organisation interne des travaux des institutions n'interdit pas cependant qu'ils puissent conférer des droits aux particuliers, dont ils peuvent se prévaloir devant le juge ${ }^{25}$. Une solution inverse aurait vidé de son sens le principe d'accès du public aux documents. En effet en dernière instance, c'est au juge qu'il doit revenir d'apprécier des conditions de la diffusion.

38 Toutefois, pour d'autres organes de la Communauté, cette sécurité juridique n'est pas garantie. En effet, s'il est par exemple louable de la part de la Banque européenne d'investissement d'avoir publié des «Règles relatives à l'accès du public aux documents 
adoptés par le Comité de direction» le 26 mars 1997 qui lui sont propres ${ }^{26}$, l'indétermination de leur autorité juridique contrebalance la bonne intention. En outre le sceaux de la confidentialité change en fonction de la nature de l'organe. Donc émergent des régimes particuliers d'accès à l'information, à côté d'un régime général applicable au triangle institutionnel de répartition du pouvoir politique dans la Communauté. Les récentes dénégations contre la Banque centrale européenne en sont une illustration supplémentaire. Son indépendance statutaire ne doit pas rimer avec isolement; isolement qui serait la conséquence d'une culture du secret. Mais l'impératif d'efficacité vient contrebalancer les exigences de la transparence ${ }^{27}$. Le même type de difficultés se posent pour Europol, en activité depuis le début de l'année 1999. L'activité de police ne répond à l'évidence pas au droit commun de la transparence; ce qui ne signifie pas que cela doit rester opaque comme l'a justement souligné le médiateur dans une invitation faite en ce sens le 18 mai $1999^{28}$. Dès lors, il apparaît qu'il y a un droit d'accès qui se dessine pour le triangle institutionnel concourant au pouvoir législatif sur des bases proches et une lex specialis pour certaines entités, dont les activités présentent une sensibilité particulière.

\section{Le régime juridique de l'accès aux documents détenus par les institutions}

39 Sur les bases énoncées dans le code de conduite en 1993, puis dans les règlements intérieurs, toute personne peut demander à avoir accès à n'importe quel document de la Commission ou du Conseil non publié, y compris les documents préparatoires et autre matériel explicatif, sans qu'il soit nécessaire de motiver la demande. À cet égard, la Commission a adopté le 9 mars 1999, un code de conduite pour les fonctionnaires européens qui leur impose de répondre aux demandes d'information ou de documentation qui leur sont adressées de façon appropriée et dans un délai raisonnable ${ }^{29}$. Celui-ci est particulièrement nécessaire pour concilier devoir de réserve et liberté d'expression.

40 En outre, si le droit d'accès est valablement contrebalancé par des exceptions, celles-ci doivent être interprétées restrictivement, de sorte que l'application du principe général consistant à conférer au public le plus large accès possible ne soit pas tenue en échec. De même, il ne suffit pas que l'institution constate que la demande tombe dans le champ d'une exception. Elle doit au contraire chaque fois que l'accès à des documents est sollicité mettre en balance l'intérêt du citoyen à cette demande et l'intérêt de l'institution à préserver le secret ${ }^{30}$. De telles décisions doivent être motivées, de manière à faire apparaître d'une façon claire et non équivoque le raisonnement de l'autorité communautaire ; ce qui permet au demandeur et au juge de contrôler le bien-fondé du refus. Le juge n'hésite pas à annuler les décisions de refus pour défaut de motivation suffisante $^{31}$. Force est d'ailleurs d'observer que les situations contentieuses ne sont pas rares; ce qui montre souvent une réticence des institutions à se soumettre aux règles, qu'elles ont pourtant elles-mêmes souscrites.

41 Dans ce cadre, quelques exemples de mise en œuvre des exceptions méritent attention, notamment ceux se rapportant au fait que «les institutions refusent l'accès à tout document dont la divulgation pourrait porter atteinte à la protection de l'intérêt public (sécurité publique, relations internationales, stabilité monétaire, procédures juridictionnelles, activités d'inspection et d'enquête) ». En ce qui concerne les procédures juridictionnelles, le juge communautaire a considéré que la Commission s'interdisait à bon droit de ne pas porter à la connaissance du public des informations qu'elle a données 
à un juge national ; ce qui signifie que l'exception vaut, y compris pour les procédures n'impliquant pas l'institution communautaire ${ }^{32}$. En outre, il convient de préciser que la liste n'est pas exhaustive et que le juge a considéré que divulguer l'avis du service juridique de la Commission et du Conseil sur la base juridique de la célèbre directive $n$ - 79/409 du 2 avril 1979 dite «oiseaux sauvages » est susceptible de mettre en cause la sécurité juridique et la stabilité de l'ordre juridique communautaire ; ce qui constitue une dimension de la protection de l'intérêt public et autorise à refuser la divulgation ${ }^{33}$. Cette solution apparait contradictoire avec l'exigence d'interprétation restrictive des exceptions au principe.

Deux arrêts rendus par le Tribunal de première instance, le 19 juillet 1999, marquent en revanche l'orientation du droit communautaire dans le sens de la plus grande transparence. La première décision se rapporte au Conseil de l'Union, qui avait refusé de communiquer à un Parlementaire européen un rapport sur les exportations d'armes conventionnelles au motif qu'il contenait des informations sensibles pour les relations de l'Union avec les pays tiers. Le juge tout en reconnaissant le bien fondé de l'argument se prononce toutefois pour un accès partiel au document pour ses chapitres ne comportant pas de risque se rapportant au souci avancé par le Conseil. Il met en avant la protection de l'intérêt public pour une transparence maximale à défaut d'être totale ${ }^{34}$. Dans un seconde décision se rapportant à la Commission, le juge rejette l'argument de droit de la Commission, selon lequel elle n'est pas l'auteur des informations demandées, qui émanent en réalité d'un comité spécialisé. Le juge observe que le comité, bien qu'autonome relève de la Commission et l'assiste dans ses tâches et doit donc être soumis aux règles de transparence que la Commission s'est fixée ${ }^{35}$.

Enfin, le fait d'évoquer le droit d'accès aux documents détenus par la Communauté n'interdit pas son contraire dans certaines hypothèses liées notamment aux risques inhérents à la société de l'information: la protection des données personnelles que détiennent les institutions communautaires dans le cadre de leurs travaux quotidiens fait en effet ici figure de paramètre non compressible. La problématique liée à la protection de la vie privée doit être prise en considération. 11 s'agit d'un champ qui bénéficie d'une réglementation, avec la directive $n^{\circ}$ 95/46 du 24 octobre 1995 du Conseil et du Parlement ${ }^{36}$. Mais elle se limite à définir une obligation de protection pesant sur les États membres et non sur les Institutions de la Communauté européenne ${ }^{37}$. Toutefois, dès 1995, la Commission comme le Conseil s'étaient engagés à en respecter les principes. Cette résolution est devenue une obligation avec le traité d'Amsterdam qui, par un nouvel article 286, étend la législation commune relative à la protection des personnes physiques à l'égard du traitement des données à caractère personnel aux institutions et organes de la Communauté. Il est également prévu un organe indépendant de contrôle chargé de surveiller le respect de la protection des données personnelles par l'administration communautaire. La Commission européenne a présenté sur cette base, le 14 juillet 1999, une proposition de règlement qui consacre pour les citoyens européens le droit d'accès, de rectification, de blocage et de suppression de données à caractère personnel figurant dans les fichiers tenus par les institutions communautaires. En cas de mésentente avec l'administration communautaire, la saisine de la future autorité indépendante fera office de recours. Le régime qui se met ainsi en place est proche de ce qui est applicable en France en raison de la législation informatique et liberté et de l'existence de la Commission Nationale Informatique et Liberté (CNIL, V. supra introduction). 
Quand on quitte le terrain juridique pour revenir aux aspects pratiques, il apparait que le taux de remise des documents par la Commission européenne est en augmentation pour atteindre 93,5\% en 1998 contre 91,9\% en 1997. Le nombre des recours contentieux se réduit. Les catégories de requérants les plus représentés sont dans l'ordre : les autorités politiques $(20,8 \%)$, les secteurs académiques (professeurs, étudiants, laboratoires avec $20,4 \%$ ), les lobbies (19\%), les industries (15,4\%). Le bilan ${ }^{38}$ apparaît satisfaisant $^{39}$ au plan quantitatif. En revanche la dimension qualitative ne fait pas émerger un droit exercé par le citoyen « lambda ».

En tout état de cause, il ressort de la construction actuelle qu'il existe désormais un principe général de « plus large accès possible du public aux documents » détenus par le Conseil ou la Commission, que toute exception doit être appliquée strictement et tout refus de diffusion motivé exhaustivement. Ce résultat a été validé par le traité d'Amsterdam. Depuis son entrée en vigueur, le traité CE contient un article 191A (renuméroté 255) selon lequel "Tout citoyen de l'Union et toute personne physique ou morale résidant ou ayant son siège dans un État membre a un droit d'accès aux documents du Parlement européen, du Conseil et de la Commission... ». On peut s'étonner que le principe ne soit qu'opposé qu'au triangle institutionnel de la Communauté. Rien n'est indiqué, notamment quant à la Banque centrale européenne. Le droit subjectif à l'accès aux documents n'est consacré que pour les institutions qui concourent à la législation communautaire. En revanche, on peut se réjouir d'un surcroît de précision quant aux bénéficiaires du droit d'accès : le citoyen et le résidant sont mis en avant, ce qui est tout de même plus satisfaisant que la simple référence au public dont la consistance juridique était insaisissable.

La même disposition assigne au législateur communautaire de fixer les principes généraux de l'accès aux documents détenus par les institutions communautaires, ainsi que les limites liées aux exigences d'intérêt public ou privé dans les deux ans suivant l'entrée en vigueur du traité d'Amsterdam, soit avant mai 2001. Des orientations générales sont à cette fin tracées par la déclaration $n^{\circ} 35$ annexée au traité sur l'Union, selon laquelle un État membre peut « demander à la Commission ou au Conseil de ne pas communiquer à des tiers un document émanant de cet État sans l'accord préalable de celui-ci »; disposition qui semble raisonnable eu égard au fait que la Communauté n'est pas ici pleinement propriétaire de l'information.

7 Il est pour le moment prématuré de se risquer à des pronostics sur la future législation. Un certain nombre de revendications sont toutefois déjà connues : la transparence doit viser tous les organes et toutes les procédures administratives, l'accès étendu aux citoyens des pays susceptibles d'adhérer, la tenue de registres publics consultables dans les États membres, la célérité du traitement de la demande d'information, etc...

En somme, né d'un assemblage composite de textes d'autorités diverses, le droit d'accès aux documents détenus par la Communauté va prochainement devenir un principe législatif. Les travaux en ce sens ont débuté avec la préparation par la Commission de deux communications se rapportant respectivement à l'accès du public aux documents et l'application du principe général de la transparence. Ces instruments auront vocation à créer les conditions d'un débat ouvert, pour l'élaboration d'une législation dans la transparence... 


\section{L'accessibilité pour le public de l'information communautaire}

49 La clarté et l'intelligibilité de la réglementation, et plus largement du droit, apparait comme une exigence démocratique. En effet, peut-on dire qu'il y a État de droit quand la règle devient inopposable à l'État car incompréhensible pour le citoyen? Cultiver l'hermétisme de la règle et donc la confidentialité de son utilisation entretient le déficit démocratique. Il n'en va pas autrement de la Communauté européenne. Si, en effet, on lui reproche souvent son caractère non démocratique, c'est en grande partie en raison de l'inaccessibilité de sa législation, jugée compliquée et incompréhensible, car touffue avec des textes trop nombreux et contradictoires. C'est pourquoi, l'effort de la Communauté porte depuis le début des années quatre-vingt-dix sur l'amélioration de la lisibilité et la codification.

\section{La lisibilité du droit}

Personne ne conteste qu'il y a depuis Maastricht un problème de repérage dans la construction européenne pour le citoyen. La sémantique apparait confuse. Au sommet de cette confusion, figure la distinction entre Union européenne et Communauté européenne. Beaucoup croient que la deuxième formulation a disparu avec l'arrivée de la première. Ce qui est erroné puisque l'Union européenne est un vocable qui désigne des réalités distinctes: $\mathrm{CE}, \mathrm{CECA}, \mathrm{CEEA}, \mathrm{PESC}, \mathrm{JAI}^{40}$. Ces réalités désignées par des sigles parfois ésotériques recouvrent des mécanismes complémentaires de réalisation de l'Union européenne. Reconnaissons que ces subtilités sont loin d'être assimilées par le citoyen communautaire, en grande partie en raison d'un défaut de lisibilité des traités. C'est pourquoi, un effort a été entrepris pour restaurer ou instaurer une meilleure orientation dans les traités comme dans la législation.

\section{La lisibilité des traités}

\section{Les apports du traité d'Amsterdam}

51 Les modifications apportées aux traités se rapportant à l'Union européenne depuis Rome le 25 mars 1957 ont été nombreuses et empreintes d'un certain empirisme. Des dispositions se maintiennent alors qu'elles sont devenues sans objet, d'autres se surajoutent par une logique alphabétique se combinant à une logique numérique, tant et si bien qu'il devenait difficile de s'orienter dans les traités. C'est pourquoi, le traité d'Amsterdam consacre sa deuxième partie à la "Simplification ». Il en découle la suppression de ce qui est caduque, l'adaptation de certaines dispositions et surtout, en raison de l'article 12 une complète renumérotation des traités. La lecture devrait devenir plus aisée. Il convient toutefois d'être prudent.

En effet, comment éviter le risque de confusion entre la version d'un article avant l'entrée en vigueur du traité d'Amsterdam le $1^{\mathrm{er}}$ mai 1999, et celle postérieure à cette date ? La Cour de justice a mis au point à cet égard une méthode de citation ${ }^{41}$ qui, il faut le reconnaitre, ne peut être comprise que par des experts de la construction européenne ; ce qui ne peut que contribuer à l'hermétisme et l'illisibilité. En outre, la compréhension de l'Union européenne ne se limite pas à lire les traités. Elle suppose l'exploration de textes 
nombreux se rapportant à des dispositions particulières. Les consulter impliquera que soit maîtrisée l'ancienne numérotation. Dès lors, au moins pour l'immédiat, l'accès à la Communauté nécessitera la maîtrise des deux numérotations, ainsi que de l'équivalence entre celles-ci. Est-ce un progrès en termes de lisibilité ?

\section{Après Amsterdam}

Le traité d'Amsterdam et ses réformes n'ont pas épuisé le thème de la lisibilité. Le rapport des sages rendu le 18 octobre 1999 relatif à la prochaine révision du fonctionnement de l'Union européenne se prononce pour une restructuration du traité, de manière à limiter le texte aux valeurs essentielles de l'Union. Son compactage servirait la garantie à sa meilleure lisibilité. Il conviendrait donc de toiletter le traité pour en retirer toute forme de règles de procédures qui viennent polluer sa compréhension. Mais il faut comprendre qu'une telle simplification comporte des conséquences juridiques importantes en raison du fait que la valeur de certaines règles va se «déconventionnaliser ». De même, une réduction du texte fondatif aux valeurs essentielles mérite-t-elle de rester du pouvoir des États ou n'y a-t-il pas ici les bases d'un projet constitutionnel pour l'Union européenne?

\section{La lisibilité de la législation}

La réglementation communautaire est souvent présentée comme mystérieuse, inaccessible pour le profane. Ce n'est pas un phénomène propre à la Communauté, mais celle-ci y est particulièrement exposée en raison de deux facteurs aggravants. En premier lieu les textes arrêtés sont le produit de compromis interétatiques et débouchent sur des métissages juridiques, qui rompent avec certaines traditions nationales. En second lieu, il convient de souligner l'évidence, en rappelant la diversité des langues nationales et les efforts de traduction nécessaires. En conséquence, l'effort de lisibilité est à la fois une exigence encore plus impérieuse que dans les autres systèmes, mais également plus difficile.

Par la déclaration n 39 annexée au traité sur l'Union européenne, l'accent est mis sur la qualité rédactionnelle de la législation communautaire. Elle rejoint ainsi la résolution du Conseil du 8 juin 1993, qui fixe quelques lignes directrices dont le «but est de rendre la législation communautaire aussi claire, simple, concise et compréhensible que possible $»^{42}$ . On note avec intérêt que doit être évité l'emploi abusif d'abréviations, du «jargon communautaire » ou de phrases trop longues. De même, la structure des textes doit être unifiée, les références à d'autres textes doivent être précises et peu nombreuses, etc. La standardisation est mise ici au service de la transparence.

Cet ensemble de prescriptions est toutefois réuni dans un document ne dépassant pas le stade de la déclaration d'intention limitée à une Institution. C'est pourquoi, la déclaration annexée au traité d'Amsterdam relance la thématique en proposant que les lignes directrices soient arrêtées d'un commun accord par le Parlement, la Commission et le Conseil, pour améliorer la qualité rédactionnelle de la législation et que ce souci soit pris en considération lors de l'examen des projets et autres propositions.

57 L'accord interinstitutionnel sur la qualité rédactionnelle de la législation communautaire ainsi programmé a été approuvé le 22 décembre $1998^{43}$. Il se présente comme une extension aux autres institutions des acquis de la résolution du Conseil du 8 juin 1993. L'accent est mis sur la normalisation et la standardisation des actes législatifs, avec une structure type, pour le préambule, les considérants, le dispositif réglementaire et 
éventuellement les annexes. Les institutions s'engagent également à éviter les phrases trop longues ou les abréviations. Enfin, les actes modifiant des textes déjà en vigueur devront être totalement réécrits, et non plus se contenter de modifier des membres de phrases, voir des mots, et les anciens textes devront être explicitement abrogés au journal officiel. Un guide pratique commun aux trois organisations est en cours de rédaction pour concrétiser les lignes directrices. Enfin, l'article 19 du nouveau règlement intérieur du Conseil du 31 mai 1999 (V. supra) fait obligation au service juridique du Conseil de vérifier la qualité rédactionnelle des propositions et projets d'actes conformément à l'accord du 22 décembre 1998.

58 Accusée régulièrement depuis le début des années quatre-vingts de trop légiférer, la Communauté semble aujourd'hui entrer dans une doctrine du «mieux légiférer ». Cela d'autant plus que la poursuite de l'effort d'accessibilité ainsi ébauché est conforté par un recours de plus en plus systématique à la codification.

\section{La codification}

59 La codification peut revêtir différentes finalités. Elle peut, en premier lieu, intervenir pour rassembler différents textes épars se rapportant à un même objet en un même document qui remplace l'ensemble avec la même autorité. Cela permet de corriger l'inaccessibilité causée par la dispersion. On parle de codification horizontale. En second lieu, la codification peut viser à intégrer à un texte donné toutes les modifications qui lui ont été apportées, de manière à conférer à sa lecture une unité qui a disparu. Cette technique peut être désignée par le terme de consolidation ou de codification verticale. Le premier point commun se situe dans l'abondance: soit l'abondance de règles se rapportant à un même objet, soit l'abondance de modifications apportées à un texte. L'autre point commun réside dans le fait de retrouver la règle de droit, la mettre ou la remettre en ordre.

60 Cet impératif de codification est devenu communautaire. L'accord interinstitutionnel du 20 décembre $1994^{44}$ le définit par " procédure qui vise à abroger les actes faisant l'objet de la codification et à les remplacer par un acte unique qui ne comporte aucune modification de la substance desdits actes ». La porte est ouverte aux deux types de codification. Toutefois, l'expérience montre une prédominance de la consolidation sur la codification horizontale ${ }^{45}$; il n'existe pas de code communautaire se rapportant à une matière donnée. En outre, il convient de souligner qu'il s'agit d'une codification à « droit constant». Elle ne doit comporter aucune modification. La codification ne doit pas être prétexte à modifier les textes. Ce préalable acquis, la codification peut suivre une voie législative accélérée.

61 Le chantier en cours repose sur l'adoption et la mise en œuvre d'une méthode de travail accélérée pour réaliser la codification officielle ${ }^{46}$, qui a été saluée au plus haut niveau par la déclaration $n^{\circ} 39$ annexée au traité sur l'Union depuis Amsterdam, qui insiste sur le fait que la Commission, le Conseil, le Parlement européen «devraient ne ménager aucun effort pour accélérer la codification officielle des textes législatifs ». Le bilan de la mise en œuvre entre 1995 et 1999 fait apparaître que près de 435 consolidations ont été réalisées, permettant de fusionner quelques 3000 actes $^{47}$.

On observe que si les déclarations d'intention sont nettes pour faire de la codification une ardente obligation, il n'en demeure pas moins qu'il n'y a pas ici une obligation juridique énoncée dans le traité. L'exigence n'est pas imposée aux institutions. Elle est fortement 
souhaitée. En conséquence, il n'existe pas de procédure propre à la codification. L'accord interinstitutionnel de 1994 ne favorise qu'une accélération de la procédure de droit commun d'adoption des textes réglementaires.

63 La codification, en l'état actuel de ses ambitions et de ses résultats, contribue imparfaitement à l'accessibilité. On est loin des grands mouvements de codification, comme celui que connaît la France depuis 1989, avec la création de la Commission supérieure de codification. Il n'existe pas de refonte ou de modernisation du droit communautaire, retracée dans des codes se rapportant à des matières données.

64 Toutefois le droit communautaire étant un droit qui s'intègre dans les ordres juridiques nationaux, on pourrait aussi poser la question de sa prise en considération dans les mouvements nationaux de codification. On peut observer, à cet égard, que le troisième tome du code de l'aviation civile rassemble la plupart des textes communautaires se rapportant à la matière. L'accessibilité du droit communautaire n'apparaît donc pas uniquement comme une question communautaire.

Cette observation nous amène à conclure plus généralement que la transparence de la Communauté européenne repose aussi sur la transparence que ses États membres veulent bien lui conférer. Mais, sur la base d'un processus de feed-back, les avancées obtenues au niveau communautaire peuvent aussi s'imposer au sein des États et contribuer à leur maturation démocratique. De même aujourd'hui, la Communauté devient le défenseur de la transparence sur les niveaux organisationnels qui la dépassent, telle l'OMC. Dès lors, théâtre de l'ascendance du principe de transparence l'Union européenne en devient aussi le promoteur.

\section{Conclusion}

Dans les deux déclinaisons retenues du principe de transparence, accès du public à l'information et accessibilité pour le public de l'information, le droit de la Communauté européenne apparait en progrès constant. Mais l'hypothèse suivant laquelle la transparence n'est jamais parfaite et qu'elle demeure sous l'emprise d'une logique asymptotique n'est pas démentie. Cette conclusion n'est pas propre à l'Union européenne. Elle reste valable pour toute forme d'organisation administrative. En revanche, on peut se féliciter de la célérité avec laquelle la transparence est devenue un impératif de la construction européenne et de la manière dont elle est aujourd'hui consolidée par son inscription dans le pacte constitutif de l'Union.

\section{NOTES}

1. Bien évidemment, il serait naïf de croire que le seul défaut d'information et de communication explique cette situation. Il apparaît en revanche pertinent d'y voir un facteur explicatif déterminant.

2. La transparence est une notion polysémique. Elle a sa signification propre dans la science de l'information et de la communication. Elle y revêt une forme asymptotique. C'est aussi un 
principe juridique qui a pour objet d'améliorer la relation entre une organisation administrative et ses usagers. Il reste donc à déterminer en quoi l'emprise du droit permet à la l'Union européenne de tendre vers cette transparence.

3. Blanchet Thérèse, "Transparence et qualité de la législation ", Revue trimestrielle de droit européen, n 33(4), 1997, pp. 915-928.

4. Sadran Pierre, «Le miroir sans tain. Réflexions sur la communication entre l'administration et les administrés ", in religion société et politique. Mélanges en hommage à Jacques Ellul, PUF 1983, pp. 797-807.

5. Communication de la Commission au Conseil, au Parlement européen, et au Comité économique et social du 2 juin 1993, «Transparence dans la Communauté », JOCE n C 166 du 17 juin 1993, p. 4.

6. «Union européenne: polémique sur la politique d'information», Europolitique $\mathrm{n}^{\circ} 2378$, 30 janvier 1999, p. 11.

7. JOCE $n^{\circ}$ L147 du 12 juin 1999, p. 13

8. JOCE $n^{\circ} \mathrm{C} 156$ du 8 juin 1993, p. 5.

9. JOCE $n^{\circ}$ C23, 23 janvier 1997, p. 1.

10. CJCE 7 juin 1983, Musique diffusion française, Pionneer et Melchers cl. Commission aff. 100 à 104/80, Rec. 1827

11. CJCE 6 avr. 1995, BPB Industries, aff. 310/93, Rec. I.

12. CJCE 18 mai 1982, AMS Europe c/. Commission, aff. 155/79, Rec. 1575.

13. CJCE 13 févr. 1979, Hoffman Laroche, aff. 85/76, Rec. 461.

14. TPI 18 sept. 1996, Postbank c/. Commission.

15. JOCE $n^{\circ}$ L158 du 23 juin 1990, p. 56.

16. CJCE, 17 juin 1998, Wilhelm Mecklenburg, aff. 321/96, Rec. I.

17. Lafay Frédérique, «L'accès aux documents du Conseil de l'Union européenne : contribution à une problématique de transparence en droit communautaire", Revue trimestrielle de droit européen, $\mathrm{n}^{\circ} 33$ (1), 1997, p. 37.

18. Code de conduite concernant l'accès du public aux documents du Conseil et de la Commission, JOCE n L340 du 31 déc. 1993, p. 41.

19. JOCE $n^{\circ} \mathrm{L} 340$ du 31 déc. 1993, p. 43.

20. JOCE n ${ }^{\circ}$ L46 du 18 févr. 1994, p. 58.

21. JOCE $n^{\circ} \mathrm{C} 67$ du 4 mars 1994, p. 5.

22. JOCE $n^{\circ}$ L263 du 25 sept. 1997, p. 27.

23. JOCE $n^{\circ}$ L339 du 10 déc. 1997, p. 18 et nº L351 du 23 déc. 1997, p. 70.

24. On relève simplement que le refus de communiquer peut être la conséquence de la protection du secret du délibéré pour les travaux des groupes politiques et des organes de l'institution siégeant ou travaillant à huis-clos.

25. CJCE 30 avril 1996, Royaume des Pays-Bas cl. Conseil de l'Union européenne, aff. 58/94, Rec. I-2169 et TPI 5 mars 1997, WWF UK. Commission, aff. 105/95, Rec. II-313.

26. JOCE $n^{\circ}$ C243 du 9 août 1997, p. 13.

27. Ricard Philippe, «En dépit d'efforts de transparence, la Banque centrale européenne ne convainc pas ", Le Monde Economie, $1^{\mathrm{er}}$ juin 1999, p. II.

28. Europolitique $\mathrm{n}^{\circ} 2408,19$ mai 1999, p. 1-3.

29. «Commission: code de conduite des fonctionnaires », Europolitique $n^{\circ} 2389,10$ mars 1999, p. I-1.

30. TPI 19 1995, John Carvel et Guardian Newspapers Ltd c/. Conseil de l'Union européenne, aff. 105/95, Rec. II-2765.

31. TPI 6 févr. 1998, Interporc Im-und Export Gmbh cl. Commission des Communautés européennes, aff. 124/96, Rec. II-231. 
32. C'est au juge national de divulguer l'information, TPI 19 mars 1998, Gérard Van der Wal, aff. $83 / 96$

33. TPI, ord., 3 mars 1998, Hanne Norup Carlsen, aff. 610/97.

34. Aff. 14/98, Heidi Hautala. Conseil de l'Union européenne.

35. Aff. 188/97, Rothmans International BV.Commission des Communautés européennes,

36. JOCE $n^{\circ}$ L281, du 23 nov. 1995, p. 31.

37. Bischoff Pierre, "L'Union européenne et la protection des données, la société de l'information à l'épreuve des droits de l'homme, Revue du Marché Commun et de l'Union européenne, $n^{\circ} 421$, sept. 1998, pp. 537-543.

38. Bilan statistique publié par la Commission le 18 mars 1999, Europolitique $n^{\circ} 2393$, du 24 mars 1999, p. 1-7.

39. Le rapport annuel du médiateur présenté le 15 avril 1999 se félicite des progrès accomplis par les diverses institutions dans l'adoption de règles internes su l'accès du public à leurs documents

40. Successivement: Communauté européenne, Communauté européenne du charbon et de l'acier, Communauté européenne à l'énergie atomique, Politique étrangère et de sécurité commune, Justice et affaires intérieures.

41. « Nouveau système de citation des dispositions des traité à la Cour ", Europolitique $\mathrm{n}^{\circ} 2430,1^{\mathrm{er}}$ sept. 1999, p. I-1.

42. JOCE $n^{\circ} \mathrm{C} 166$ du 17 juin 1993, p. 1.

43. JOCE $n^{\circ} \mathrm{C}$ du 17 mars 1999.

44. Accord interinstitutionnel instituant une méthode accélérée en vue d'une codification officielle des textes législatifs, JOCE ${ }^{\circ} \mathrm{C} 102$ du 4 avril 1996, p. 2.

45. Dragone Stéfania, «La codification communautaire : techniques et procédures », Revue du Marché Unique Européen, $n^{\circ} 1 / 98$, pp. 77-94.

46. JOCE $n^{\circ} \mathrm{C} 102$ du 4 avril 1996, p. 2.

47. Rapport de la Commission européenne « Mieux légiférer », $1^{\mathrm{er}}$ déc. 1998.

\section{RÉSUMÉS}

Réduire la distance entre l'administré et l'administration est une préoccupation du droit français, depuis la fin des années soixante-dix, résolue en grande partie, par la mise en œuvre du principe de transparence. Ce principe concerne aujourd'hui la Communauté européenne sujette à de nombreuses critiques visant à dénoncer son caractère confidentiel, qui alimente un déficit démocratique. Le droit a donc évolué, d'abord pour garantir un accès amélioré à l'information sur la Communauté, ainsi qu'à l'information détenue par celle-ci. L'objectif de la réalisation du marché intérieur, puis le traité de Maastricht ont engendré un surcroît de transparence sur ces deux points. On observe toutefois qu'il n'existe pas de législation générale. L'ouverture de l'Union sur ses citoyens repose encore sur un certain empirisme. Le traité d'Amsterdam, en vigueur depuis mai 1999, est à cet égard annonciateur de progrès, notamment par la consécration du principe général de transparence, comme modalité inhérente à la réalisation de l'Union européenne

Since the end of the 1970s the principle of «transparency ", the effort to clarify administrative dealings, was established in french law in order to bring the centralised state doser to its citizens. 
This principle is now established in the European Union as a reaction to criticism regarding the opacity of an organisation not known for scrupulous observance of democratic precepts. However, the idea of bringing Europe closer to its citizens in terms of access to information, held by or regarding the Community, has been applied in an experimental fashion. But the Treaty of Amsterdam now makes «transparency » a general principle of European law.

INDEX

Mots-clés : transparence, Communaute Européenne, Union Européenne, droit à l'information, codification, lisibilité, accessibilité, secret, confidentialité, diffusion, publicité

\section{AUTEUR}

\section{LOÏC GRARD}

Professeur de droit public. Université Montesquieu Bordeaux IV. Enseignements en droit communautaire et en droit international. En 1996, il a publié Droit communautaire des transports aériens, Joly, et Vers un service public européen, Aspe éditions. Et, en 1998, à La documentation française, « La Communauté européenne et le développement des départements d'outre-mer ». Ainsi que plusieurs articles sur la construction de l'Union européenne et la réalisation du marché intérieur. 